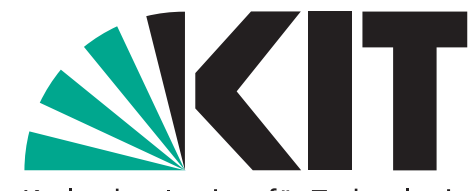

Karlsruher Institut für Technologie

\title{
Non-bossy social classification
}

by Dinko Dimitrov and Clemens Puppe

No. 23 | APRIL 2011

\section{WORKING PAPER SERIES IN ECONOMICS}

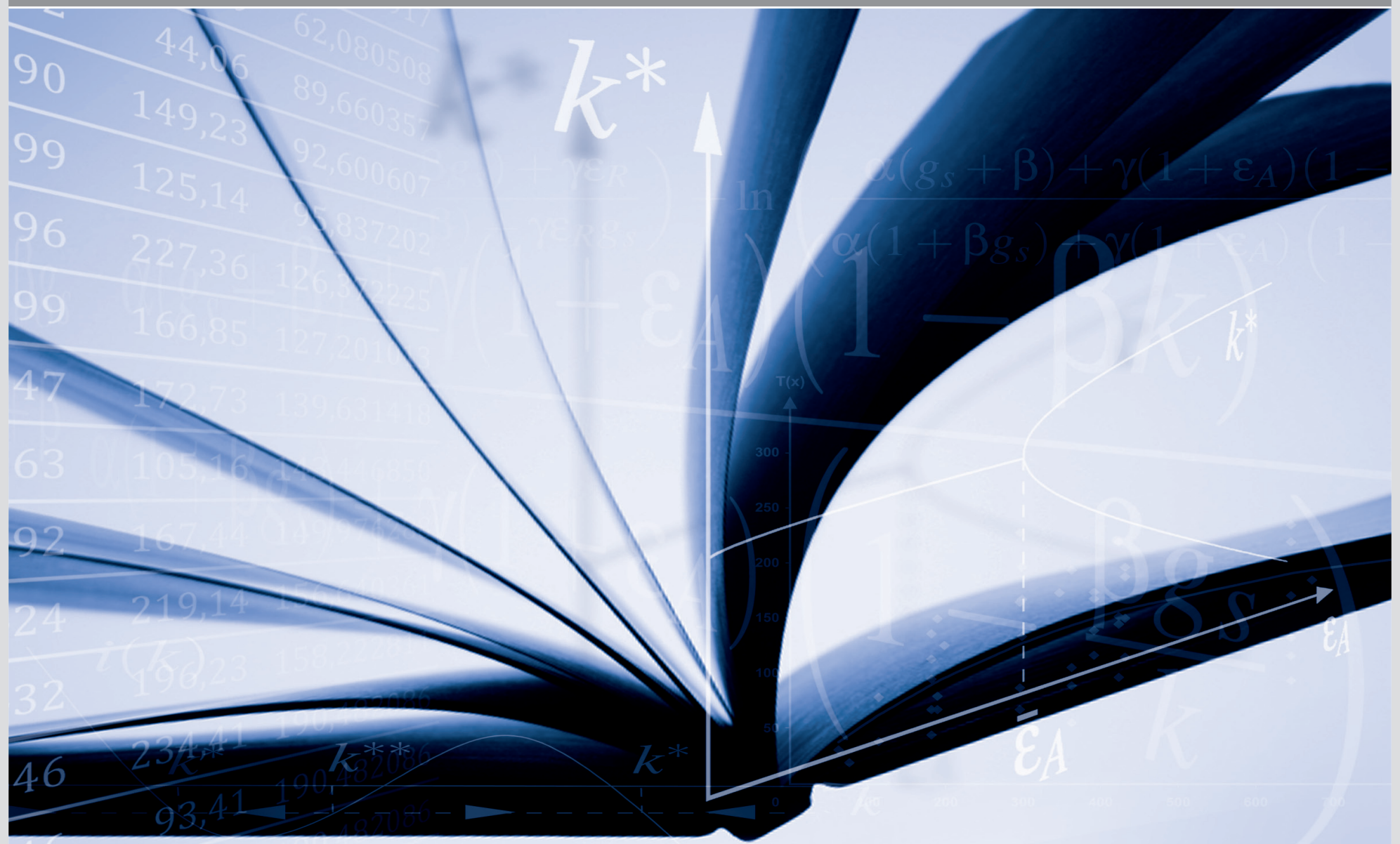




\section{Impressum}

Karlsruher Institut für Technologie (KIT)

Fakultät für Wirtschaftswissenschaften

Institut für Wirtschaftspolitik und Wirtschaftsforschung (IWW)

Institut für Wirtschaftstheorie und Statistik (ETS)

Schlossbezirk 12

76131 Karlsruhe

KIT - Universität des Landes Baden-Württemberg und nationales Forschungszentrum in der Helmholtz-Gemeinschaft

Working Paper Series in Economics

No. 23, April 2011

ISSN 2190-9806

econpapers.wiwi.kit.edu 


\title{
Non-bossy social classification*
}

\author{
Dinko Dimitrov ${ }^{\dagger} \quad$ Clemens Puppe ${ }^{\ddagger}$
}

April 10, 2011

\begin{abstract}
We consider the problem of how societies should be partitioned into classes if individuals express their views about who should be put with whom in the same class. A non-bossy social aggregator depends only on those cells of the individual partitions the society members classify themselves in. This fact allows us to concentrate on a corresponding "opinion graph" for each profile of views. By means of natural sovereignty, liberalism, and equal treatment requirements, we characterize the non-bossy aggregators generating partitions in which the social classes are refinements of the weakly connected components of the opinion graph.
\end{abstract}

JEL Classification: D71

Keywords: social aggregation, group identity, liberalism, non-bossiness

\section{Introduction}

In this paper we study the formation of groups or classes from a social choice perspective. Adapting the framework of Fishburn and Rubinstein (1986), we

*This paper was partly written while the first author was visiting the Delhi Center of the Indian Statistical Institute. The hospitality of the Institute and the helpful comments and suggestions of Anirban Kar, Debasis Mishra, and Arunava Sen are gratefully acknowledged.

$\dagger$ Chair of Economic Theory, Saarland University, Germany; e-mail: dinko.dimitrov@mx.uni-saarland.de

${ }^{\ddagger}$ Chair of Economic Theory, Karlsruhe Institute of Technology, Germany; e-mail: clemens.puppe@kit.edu 
consider an environment in which every individual has a view about how the society should be partitioned into classes. A group identity function assigns then to each profile of views a societal decomposition into classes. In the aggregation problem considered here the number of classes is endogenously determined. This is in contrast to environments in which the number of social groups is assumed to be fixed and their names matter (cf. Çengelci and Sanver 2010, Dimitrov et al. 2007, Houy 2007, Kasher and Rubinstein 1997, Miller 2008, Samet and Schmeidler 2003, among others).

The most studied rule in the context of aggregating partitions is the conjunctive aggregator which classifies two individuals in the same social group if and only if everyone in the society thinks so. This function belongs to the class of rules characterized by Fishburn and Rubinstein (1986) in the context of the aggregation of equivalence relations (see also Mirkin 1975, Barthélemy et al. 1986, Barthélemy 1988, Dimitrov et al. 2011) and it was recently axiomatized by Houy (2007) in the context of group identification. The central axiom in most of these characterizations is a binary independence condition requiring the decision of whether or not two individuals belong to the same social class to depend only on the individual classifications with respect to these two individuals.

By contrast, we concentrate in this paper on non-bossy social aggregation which requires the group identity function to depend only on one cell from the individual partition of each society member - namely on the cell the corresponding individual classifies himself in. Intuitively, non-bossiness thus states that the decision of whether or not two individuals belong to the same class should not depend on the view of unconcerned individuals. Put in a different way, non-bossiness makes the social aggregation dependent on the information provided only by a corresponding opinion graph on the set of individuals. A directed edge $(i, j)$ in this graph corresponds to the situation in which individual $i$ classifies himself in the same group with individual $j$. The group identity functions we introduce in this paper correspond to 
particular ways of decomposing this graph. Specifically, any group identity function satisfying a positive liberalism condition and a simple sovereignty requirement decomposes the graph into particular refinements of its weakly connected components. Having described the set of admissible partitions from which such a group identity function selects the societal classification, we then introduce natural equal treatment requirements as to narrow the admisible set and to characterize the non-bossy rule generating the coarsest admissible partition.

\section{Basic definitions and notation}

The society is denoted by $N=\{1, \ldots, n\}$, and $\Pi$ is the set of all partitions of $N$. Recall that a partition of $N$ is a collection of non-empty, pairwise disjoint subsets of $N$ whose union is $N$. We call these subsets groups or classes. A partition $\pi$ is said to refme another partition $\pi^{\prime}$, denoted by $\pi \leq \pi^{\prime}$, if every group from $\pi$ is contained in some group from $\pi^{\prime}$; we also say in this case that $\pi^{\prime}$ is coarser than $\pi$. The refinement relation is a partial ordering on $\Pi$.

For each $i \in N$, individual $i$ s view is $\pi^{i} \in \Pi$. Moreover, for each $j \in N$, we denote by $\pi_{j}^{i}$ the cell in the partition $\pi^{i}$ that contains individual $j$. For instance, if $\pi_{2}^{1}=\pi_{3}^{1}=\pi_{5}^{1}=\{2,3,5\} \in \pi^{1}$ then, according to individual 1, individuals 2, 3, and 5 should belong to the same social group. A profile of individual views is denoted by $\pi:=\left(\pi^{1}, \ldots, \pi^{n}\right) \in \Pi^{N}$. For $i \in N$ and $\pi^{\prime i} \in \Pi$, we write $\left(\pi^{-i}, \pi^{\prime i}\right)$ to denote the profile at which $i$ s view $\pi^{i}$ is replaced by $\pi^{\prime i}$. Moreover, $\lambda_{N}$ denotes the partition of $N$ into singletons.

Let $\mathcal{D} \subseteq \Pi^{N}$ contain $\left(\lambda_{N}, \ldots, \lambda_{N}\right)$ and $(\{N\}, \ldots,\{N\})$. A group identity function on $\mathcal{D}$ is a mapping $f: \mathcal{D} \rightarrow \Pi$ which assigns to each profile $\boldsymbol{\pi} \in \mathcal{D}$ of individual views a partition $f(\boldsymbol{\pi}) \in \Pi$ of the society into social groups. For all $i \in N, f(\boldsymbol{\pi})_{i}$ is the social group to which individual $i$ belongs according to $f$. 
For any $i \in N$ and $\pi^{i} \in \Pi$, denote by $\bar{\pi}^{i} \in \Pi$ any partition such that $\bar{\pi}_{i}^{i}=\pi_{i}^{i}$. That is, $\pi^{i}$ and $\bar{\pi}^{i}$ may differ only with respect to the cells individual $i$ does not belong to.

Non-Bossiness (NB): A group identity function $f$ satisfies Non-Bossiness if for all $i \in N$, all $\boldsymbol{\pi} \in \mathcal{D}$, and all $\bar{\pi}^{i} \in \Pi, f(\boldsymbol{\pi})=f\left(\pi^{-i}, \bar{\pi}^{i}\right)$.

Non-bossiness thus requires that an individual can influence the social classification only via his individual opinion about the social group he himself belongs to. ${ }^{1}$

Note that Fishburn and Rubinsteins' conjunctive aggregator does not satisfy NB. To see this, take $\boldsymbol{\pi} \in \mathcal{D}$ to be such that $\{1,2\} \in \pi^{i}$ for all $i \in N$. Further, for some $j \in N \backslash\{1,2\}$, let $\bar{\pi}^{j}$ be such that $\{1\},\{2\} \in \bar{\pi}^{j}$. Then, the conjunctive aggregator classifies 1 and 2 together if the profile is $\boldsymbol{\pi}$, while 1 and 2 are classified as being single if the profile is $\left(\pi^{-j}, \bar{\pi}^{j}\right)$; thus, NB is violated.

As is easily verified by repeated application of NB, the non-bossiness condition restricts a group identity function to depend only on the individual views with respect to the groups the corresponding individuals themselves belong to:

Fact $A$ group identity function $f$ satisfies $N B$ if and only if $f(\boldsymbol{\pi})=f\left(\boldsymbol{\pi}^{\prime}\right)$ for all $\boldsymbol{\pi}, \boldsymbol{\pi}^{\prime} \in \mathcal{D}$ with $\pi_{i}^{i}=\pi_{i}^{\prime i}$ for all $i \in N$.

This fact allows us to look at an underlying "opinion graph" when searching for non-bossy social aggregators. Recall that a directed graph $H=(V, E)$ consists of a set of vertices $V$ and a set of directed edges $E \subseteq V \times V$. Let $X \subseteq V$. We say that $X$ is weakly connected if, for every $i, j \in X$, there is a sequence of vertices $k_{1}, k_{2}, \ldots, k_{m} \in X$ for some positive integer $m$ such that $k_{1}=i, k_{m}=j$, and either $\left(k_{\ell}, k_{\ell+1}\right) \in E$ or $\left(k_{\ell+1}, k_{\ell}\right) \in E$ for each

\footnotetext{
${ }^{1}$ Our condition is closely related to, but not a literal adaptation of, the non-bossiness condition introduced by Satterthwaite and Sonnenschein (1981) in the context of social choice functions.
} 
$1 \leq \ell \leq m-1$; if one only requires $\left(k_{\ell}, k_{\ell+1}\right) \in E$ for each $1 \leq \ell \leq m-1$, the set $X$ of vertices is called strongly connected. We call $X$ a weakly (strongly) connected component if it is weakly (strongly) connected and, for all $Y \subseteq N$ which properly contain $X, Y$ is not weakly (strongly) connected. The weak (strong) decomposition of $H$ is its (unique) decomposition into weakly (strongly) connected components. Clearly, the strong decomposition of $H$ is a refinement of its weak decomposition.

To describe the decompositions we study in the next section, we construct an opinion graph $H_{\pi}=\left(V_{\pi}, E_{\pi}\right)$ for each $\boldsymbol{\pi} \in \mathcal{D}$, where $V_{\pi}=N$ and $E_{\pi}=$ $\left\{(i, j): i \neq j, j \in \pi_{i}^{i}\right\}$. Notice again that the Fact mentioned above allows us to restrict ourselves to the information provided by $H_{\boldsymbol{\pi}}$. More precisely, the group identity functions presented in this paper assign to each profile of individual views particular refinements of the weakly connected components in $H_{\pi}$.

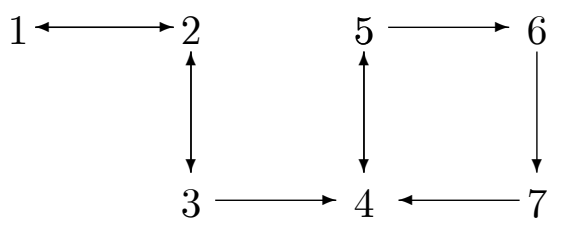

8

Figure 1: An eight-member society

Figure 1 depicts a society consisting of eight individuals. Given the above definitions, $H_{\pi}$ has two weakly connected components $-\{1,2,3,4,5,6,7\}$ and $\{8\}$, and three strongly connected components $-\{1,2,3\},\{4,5,6,7\}$, and $\{8\}$. 


\section{Weakly connected components and their refinements}

We consider two simple axioms and describe first the set of admissible partitions from which any group identity function that satisfies the two axioms selects the societal classification. We then introduce natural equal treatment requirements as to narrow the admissible set and to characterize the non-bossy rule generating the coarsest admissible partition.

The first axiom has a liberal flavor and states that the aggregator puts two individuals in the same social group provided that both individuals think that they belong together (cf. Houy 2007).

Positive Liberalism (PL): A group identity function $f$ satisfies Positive Liberalism if for every profile $\boldsymbol{\pi} \in \mathcal{D}$ and all $i, j \in N, i \in \pi_{j}^{j}$ and $j \in \pi_{i}^{i}$ imply $f(\boldsymbol{\pi})_{i}=f(\boldsymbol{\pi})_{j}$.

In order to explain our next axiom, Negative Group Sovereignty, imagine a situation in which the society $N$ is partitioned into two non-empty subsets $N_{1}$ and $N_{2}$. Consider the case in which every individual in $N_{1}$ thinks that he belongs in the same group with individuals only from $N_{1}$ and that every individual in $N_{2}$ puts himself in the same group with individuals only from $N_{2}$. Then, it seems reasonable to require that an aggregator should not classify an individual from $N_{1}$ and a second one from $N_{2}$ as being members of the same social group.

Negative Group Sovereignty (NGS): A group identity function $f$ satisfies Negative Group Sovereignty if for every profile $\boldsymbol{\pi} \in \mathcal{D}$ and for any two disjoint subsets $N_{1}$ and $N_{2}$ of $N$ with $N=N_{1} \cup N_{2}$ we have that $\pi_{i}^{i} \subseteq N_{1}$ and $\pi_{j}^{j} \subseteq N_{2}$ for all $i \in N_{1}$ and all $j \in N_{2}$ imply $f(\boldsymbol{\pi})_{i} \neq f(\boldsymbol{\pi})_{j}$ for all $i \in N_{1}$ and all $j \in N_{2}$.

As we show next, a group identity function satisfying PL and NGS nec- 
essarily selects, for each $\boldsymbol{\pi} \in \mathcal{D}$, a partition $f(\boldsymbol{\pi})$ from the set $\mathcal{R}(\boldsymbol{\pi})$ we introduce now.

The coarsest partition contained in $\mathcal{R}(\boldsymbol{\pi})$ is $\pi^{*}(\boldsymbol{\pi})$, the partition of $N$ into weakly connected components in $H_{\boldsymbol{\pi}}$. The finest partition $\pi_{*}(\boldsymbol{\pi})$ included in $\mathcal{R}(\boldsymbol{\pi})$ replaces each $D \in \pi^{*}(\boldsymbol{\pi})$ by its finest partition $\pi^{D}=\left\{D_{1}, \ldots, D_{K}\right\}$ for which the following condition is satisfied: for all $k \in\{1, \ldots, K\}$ and all $i \in D_{k}$ and $j \in D$, we have that $j \in \pi_{i}^{i}$ and $i \in \pi_{j}^{j}$ imply $j \in D_{k}$.

The set $\mathcal{R}(\boldsymbol{\pi})$ is then defined as follows:

$$
\mathcal{R}(\boldsymbol{\pi})=\left\{\pi \in \Pi: \pi_{*}(\boldsymbol{\pi}) \leq \pi \leq \pi^{*}(\boldsymbol{\pi})\right\}
$$

Thus, each social class in every partition $\pi$ in $\mathcal{R}(\boldsymbol{\pi})$ belongs to a refinement of $\{D\}$ and to a coarsening of $\left\{\pi^{D}\right\}$ for some weakly connected component $D$ in $H_{\boldsymbol{\pi}}$.

Let us have a look again at Fig. 1 and describe the way the corresponding axioms restrict the decomposition of the depicted society. First, by NGS, the eighth individual cannot be grouped in the same class with any other individual. Second, by PL, the following individuals have to be classified together: 1, 2, and 3; and 4 and 5. Hence, the coarsest partition compatible with these restrictions is $\{\{1,2,3,4,5,6,7\},\{8\}\}$, while the finest one is $\{\{1,2,3\},\{4,5\},\{6\},\{7\},\{8\}\}$. Note that the societal classification into strongly connected components $\{\{1,2,3\},\{4,5,6,7\},\{8\}\}$ is compatible with NGS and PL as well, and it is a member of the set $\mathcal{R}(\boldsymbol{\pi})$ for the problem considered in Fig. 1.

Proposition 1 A group identity function $f$ satisfies $P L$ and NGS if and only if $f(\boldsymbol{\pi}) \in \mathcal{R}(\boldsymbol{\pi})$ for all $\boldsymbol{\pi} \in \mathcal{D}$. Moreover, the two axioms are independent.

Proof. Let $f$ be such that $f(\boldsymbol{\pi}) \in \mathcal{R}(\boldsymbol{\pi})$ for all $\boldsymbol{\pi} \in \mathcal{D}$. Notice then that $f$ satisfies PL as, for all $\boldsymbol{\pi} \in \mathcal{D}$, any partition in $\mathcal{R}(\boldsymbol{\pi})$ classifies in the same group any two individuals $i$ and $j$ with $i \in \pi_{j}^{j}$ and $j \in \pi_{i}^{i}$. Suppose now that $f$ violates NGS. This implies that there is some profile $\boldsymbol{\pi}^{\prime} \in \mathcal{D}$ and a partition 
of $N$ into two non-empty subsets $N_{1}$ and $N_{2}$ with $\pi_{i}^{\prime i} \subseteq N_{1}$ and $\pi_{j}^{\prime j} \subseteq N_{2}$ for all $i \in N_{1}$ and all $j \in N_{2}$ such that $f\left(\boldsymbol{\pi}^{\prime}\right)_{k_{1}}=f\left(\boldsymbol{\pi}^{\prime}\right)_{k_{2}}$ for some $k_{1} \in N_{1}$ and $k_{2} \in N_{2}$. Notice that we have in such a case a direct contradiction to $f\left(\boldsymbol{\pi}^{\prime}\right) \in \mathcal{R}\left(\boldsymbol{\pi}^{\prime}\right)$.

Consider now a group identity function $f$ which satisfies PL and NGS, and take $\boldsymbol{\pi} \in \mathcal{D}$. In what follows we show that $f(\boldsymbol{\pi}) \notin \mathcal{R}(\boldsymbol{\pi})$ leads to a contradiction.

(1) Consider first the case in which there is $D^{\prime} \in f(\boldsymbol{\pi})$ that strictly contains some $D \in \pi^{*}(\boldsymbol{\pi})$ (and thus, $\left|D^{\prime}\right| \geq 2$ ). Define then $N_{1}:=D$ and $N_{2}:=N \backslash D$ and note that both sets are non-empty. Let $k \in D=N_{1}$ and $k^{\prime} \in D^{\prime} \backslash D \subseteq N_{2}$. Since $D \in \pi^{*}(\boldsymbol{\pi})$, we have $\pi_{i}^{i} \subseteq N_{1}$ for all $i \in N_{1}$ and $\pi_{j}^{j} \subseteq N_{2}$ for all $j \in N_{2}$. Hence, by NGS, we should have $f(\boldsymbol{\pi})_{k} \neq f(\boldsymbol{\pi})_{k^{\prime}}$ in contradiction to $f(\boldsymbol{\pi})_{k}=f(\boldsymbol{\pi})_{k^{\prime}}=D^{\prime}$.

(2) We show next that it is impossible for $f(\boldsymbol{\pi})$ to contain a social class which is a strict subset of some class from the finest partition $\pi_{*}(\boldsymbol{\pi})$ contained in $\mathcal{R}(\boldsymbol{\pi})$. If this were the case, there would exist groups $D^{\prime}, D^{\prime \prime}$ and $D^{\prime \prime \prime}$ s.t. $D^{\prime} \subset D^{\prime \prime} \in \pi^{D^{\prime \prime \prime}}$ and $D^{\prime} \in f(\boldsymbol{\pi})$. However, this would imply that we can find individuals $i \in D^{\prime}$ and $j \in D^{\prime \prime} \backslash D^{\prime}$ such that $i \in \pi_{j}^{j}$ and $j \in \pi_{i}^{i}$. By PL, $f(\boldsymbol{\pi})_{i}=f(\boldsymbol{\pi})_{j}$ in contradiction to $f(\boldsymbol{\pi})_{i}=D^{\prime}$ and $j \notin D^{\prime}$.

We conclude that $f(\boldsymbol{\pi}) \in \mathcal{R}(\boldsymbol{\pi})$.

In order to show the independence of the axioms, consider the following two rules. Each rule satisfies one of the axioms but not the other. Moreover, for each of these rules, there is a profile $\boldsymbol{\pi} \in \Pi^{N}$ s.t. $f(\boldsymbol{\pi}) \notin \mathcal{R}(\boldsymbol{\pi})$.

(not PL) Take the aggregator $f^{\prime}$ defined as follows: for all $\boldsymbol{\pi} \in \mathcal{D}, f^{\prime}(\boldsymbol{\pi})=$ $\lambda_{N}$. This aggregator clearly violates PL while satisfying NGS. We have for this rule that $f^{\prime}(\{N\}, \ldots,\{N\}) \notin \mathcal{R}(\{N\}, \ldots,\{N\})=\{\{N\}\}$.

(not NGS) Consider the aggregator $f^{\prime \prime}$ defined as follows: for all $\boldsymbol{\pi} \in$ $\mathcal{D}, f^{\prime \prime}(\boldsymbol{\pi})=\{N\}$. This rule satisfies PL but not NGS, and we have that $f^{\prime \prime}\left(\lambda_{N}, \ldots, \lambda_{N}\right) \notin \mathcal{R}\left(\lambda_{N}, \ldots, \lambda_{N}\right)=\left\{\lambda_{N}\right\}$.

This completes the proof. 
Our next condition is an equal treatment requirement. It says that all pairs of individuals in a profile such that one but only one individual of the pair puts himself/herself together in a class with the other should be treated in the same way.

Equal Treatment (ET): A group identity function $f$ satisfies Equal Treatment if for every profile $\pi \in \mathcal{D}$ and all $i, j, k, \ell \in N$, we have that $j \in \pi_{i}^{i}$, $i \notin \pi_{j}^{j}$ and $\ell \in \pi_{k}^{k}, k \notin \pi_{\ell}^{\ell}$ imply $j \in f(\boldsymbol{\pi})_{i} \Rightarrow \ell \in f(\boldsymbol{\pi})_{k}$.

Proposition 2 A group identity function $f$ satisfies PL, NGS, and ET if and only if $f(\boldsymbol{\pi}) \in\left\{\pi_{*}(\boldsymbol{\pi}), \pi^{*}(\boldsymbol{\pi})\right\}$ for all $\boldsymbol{\pi} \in \mathcal{D}$. Moreover, the three axioms are independent.

Proof. It follows from Proposition 1 that $f$ satisfies PL and NGS. As it can be easily seen, $f$ satisfies ET as well. Let now $f$ satisfy PL, NGS, and ET and take $\boldsymbol{\pi} \in \mathcal{D}$. If $\boldsymbol{\pi}$ is such that for some $X \in \pi_{*}(\boldsymbol{\pi})$ there are $i, j \in X$ with $j \in \pi_{i}^{i}$ and $j \notin \pi_{i}^{i}$, then $i \in f(\boldsymbol{\pi})_{j}$ follows by PL. ET requires then that for all $k, \ell \in N$, we have $\ell \in f(\boldsymbol{\pi})_{k}$ if $\ell \in \pi_{k}^{k}$ and $k \notin \pi_{\ell}^{\ell}$. Hence, all members of a weakly connected component belong to the same social group which, by the repeated application of NGS, gives us $\pi^{*}(\boldsymbol{\pi})$. If no $X \in \pi_{*}(\boldsymbol{\pi})$ contains two individuals with the above property, then the repeated application of ET requires that the wishes of all $i, j \in N$ with $j \in \pi_{i}^{i}$ and $j \notin \pi_{i}^{i}$ are either all granted or all denied. Thus, applying NGS repeatedly delivers either $\pi_{*}(\boldsymbol{\pi})$ (if all wishes are denied) or $\pi^{*}(\boldsymbol{\pi})$ (if all wishes are granted). Finally, the aggregators $f^{\prime}$ and $f^{\prime \prime}$ constructed in the proof of Proposition 1 serve also as examples for the independence of PL and NGS, respectively. As for our last axiom, the rule $f^{\prime \prime \prime}$ assigning to each $\pi \in \mathcal{D}$ the strong decomposition of $H_{\boldsymbol{\pi}}$ satisfies PL and NGS while violating ET.

Remark 1 One can strengthen the Equal Treatment axiom by requiring that for every profile $\pi \in \mathcal{D}$ and all $i, j, k, \ell \in N$ with $i \neq j$ and $k \neq \ell$, we have that $j \in \pi_{i}^{i}$ and $\ell \in \pi_{k}^{k}$ imply $j \in f(\boldsymbol{\pi})_{i} \Rightarrow \ell \in f(\boldsymbol{\pi})_{k}$. Call this condition $E T^{+}$; evidently, ET ${ }^{+}$implies ET. It is easy to see that NGS to- 
gether with $E T^{+}$restrict $f$ to select a partition from the set $\left\{\lambda_{N}, \pi^{*}(\boldsymbol{\pi})\right\}$ for all $\boldsymbol{\pi} \in \mathcal{D}$. More precisely, if $\boldsymbol{\pi}=\left(\lambda_{N}, \ldots, \lambda_{N}\right)$, then $f(\boldsymbol{\pi})=\lambda_{N}$ follows by $N G S$; and if $i \in \pi_{j}^{j}$ for some $i, j \in N$ with $i \neq j$, then $E T^{+}$can be satisfied either if $i \in f(\boldsymbol{\pi})_{j}$ holds for all such pairs or for none of them. Hence, $f(\boldsymbol{\pi}) \in\left\{\lambda_{N}, \pi^{*}(\boldsymbol{\pi})\right\}$ follows by the additional application of NGS.

Remark 2 Not every group identity function satisfying PL, NGS, and ET (or $E T^{+}$) is non-bossy. Consider for instance a society of three members having the views $\pi^{1}=\{\{1,2\},\{3\}\}, \pi^{2}=\pi^{3}=\{\{1\},\{2\},\{3\}\}$, and let $\pi=\{\{1,2\},\{3\}\}$ and $\pi^{\prime}=\{\{1\},\{2\},\{3\}\}$. Defme a group identity function $f$ as follows: $f$ selects $\pi$ if the individual views w.r.t. their own classification are as above and individual 3 thinks that individuals 1 and 2 belong together, and $f$ selects $\pi^{\prime}$ if the individual views w.r.t. their own classification are as above and individual 3 does not think that individuals 1 and 2 belong together, and $f$ selects the partition into weakly connected components in any other case. Clearly, $f$ then satisfies PL, NGS, and both versions of ET but violates non-bossiness.

The following condition strengthens the Equal Treatment requirement by imposing it not only within but also across profiles.

Strong Equal Treatment (SET): A group identity function $f$ satisfies Strong Equal Treatment if for all $\boldsymbol{\pi}, \boldsymbol{\pi}^{\prime} \in \mathcal{D}$ and all $i, j, k, \ell \in N$, we have that $j \in \pi_{i}^{i}, i \notin \pi_{j}^{j}$ and $\ell \in \pi_{k}^{\prime k}, k \notin \pi_{\ell}^{\prime \ell}$ imply $j \in f(\boldsymbol{\pi})_{i} \Rightarrow \ell \in f\left(\boldsymbol{\pi}^{\prime}\right)_{k}$.

Proposition 3 A group identity function $f$ satisfies $P L, N G S$, and SET if and only if for all $\boldsymbol{\pi} \in \mathcal{D}$ we have either $f(\boldsymbol{\pi})=\pi_{*}(\boldsymbol{\pi})$ or $f(\boldsymbol{\pi})=\pi^{*}(\boldsymbol{\pi})$. Moreover, the three axioms are independent and together imply non-bossiness.

Proof. Let $f$ satisfy these three axioms. By Proposition 2, $f(\boldsymbol{\pi}) \in$ $\left\{\pi_{*}(\boldsymbol{\pi}), \pi^{*}(\boldsymbol{\pi})\right\}$ holds for all $\boldsymbol{\pi} \in \mathcal{D}$. Let $\boldsymbol{\pi}$ and $\boldsymbol{\pi}^{\prime}$ be two different profiles such that $\pi_{*}(\boldsymbol{\pi}) \neq \pi^{*}(\boldsymbol{\pi})$ and $\pi_{*}\left(\boldsymbol{\pi}^{\prime}\right) \neq \pi^{*}\left(\boldsymbol{\pi}^{\prime}\right)$. In order to prove our statement it suffi ces to show that $f(\boldsymbol{\pi})=\pi_{*}(\boldsymbol{\pi})$ and $f\left(\boldsymbol{\pi}^{\prime}\right)=\pi^{*}\left(\boldsymbol{\pi}^{\prime}\right)$ lead 
to a contradiction. Note first that $f\left(\boldsymbol{\pi}^{\prime}\right)=\pi^{*}\left(\boldsymbol{\pi}^{\prime}\right) \neq \pi_{*}\left(\boldsymbol{\pi}^{\prime}\right)$ implies $j \in \pi_{i}^{\prime i}$ and $i \notin \pi_{j}^{\prime j}$ for some $i, j \in N$; moreover, $j \in f\left(\boldsymbol{\pi}^{\prime}\right)_{i}$. On the other hand, $f(\boldsymbol{\pi})=\pi_{*}(\boldsymbol{\pi}) \neq \pi^{*}(\boldsymbol{\pi})$ implies the existence of $k, \ell \in N$ with $\ell \in \pi_{k}^{k}, k \notin \pi_{\ell}^{\ell}$, and $\ell \notin f(\boldsymbol{\pi})_{k}$. Hence, we have a violation of SET. Note finally that the aggregators $f^{\prime}, f^{\prime \prime}$, and $f^{\prime \prime \prime}$ constructed in the proofs of Propositions 1 and 2 serve also as examples for the independence of PL, NGS, and SET, respectively. The non-bossiness condition is then trivially satisfied as for all $\boldsymbol{\pi} \in \mathcal{D}$ we have either $f(\boldsymbol{\pi})=\pi_{*}(\boldsymbol{\pi})=\pi_{*}\left(\pi^{-i}, \bar{\pi}^{i}\right)$ or $f(\boldsymbol{\pi})=\pi^{*}(\boldsymbol{\pi})=\pi^{*}\left(\pi^{-i}, \bar{\pi}^{i}\right)$.

Proposition 4 Let $n \geq 3$ and $f$ be a group identity function defined on the unrestricted domain $\mathcal{D}=\Pi^{N}$. Then $f$ satisfies $P L, N G S$, and SET if and only if $f(\boldsymbol{\pi})=\pi^{*}(\boldsymbol{\pi})$ for all $\boldsymbol{\pi} \in \Pi^{N}$.

Proof. Let $\boldsymbol{\pi} \in \mathcal{D}$ be such that $\pi_{i}^{i}=\{i, j\}, \pi_{j}^{j}=\{i, j, k\}$, and $\pi_{k}^{k}=\{i, j, k\}$ for $i, j, k \in N$. By twofold application of PL, $k \in f(\boldsymbol{\pi})_{i}$, hence by SET $f(\boldsymbol{\pi})=\pi^{*}(\boldsymbol{\pi})$. The additional use of SET results then in $f(\boldsymbol{\pi})=\pi^{*}(\boldsymbol{\pi})$ for all $\pi \in \Pi^{N}$.

Remark 3 As the proof of Proposition 4 shows, axioms PL, NGS and SET characterize the non-bossy aggregation rule $f^{*}$ defined by $f^{*}(\boldsymbol{\pi})=\pi^{*}(\boldsymbol{\pi})$ for all $\boldsymbol{\pi} \in \mathcal{D}$ provided that the domain $\mathcal{D}$ contains a profile $\boldsymbol{\pi} \in \Pi^{N}$ s.t. for some $X \in \pi_{*}(\boldsymbol{\pi})$ there are $i, j \in X$ with $j \in \pi_{i}^{i}$ and $i \notin \pi_{j}^{j}$.

\section{Discussion and conclusion}

This paper is devoted to the study of group identification problems in which the decision of whether or not two individuals belong to the same class does not depend on the view of unconcerned individuals. In this setup, a graph representation of the individual views allowed us to introduce new group identity functions and to characterize them in terms of appropriate (positive) liberalism and (negative) sovereignty axioms. 
It is natural to ask whether characterization results for non-bossy group identity functions can be obtained if one uses a negatively formulated liberalism axiom and a positive counterpart of the negative sovereignty condition. Specifically, consider the following negative liberalism axiom introduced in Houy (2007). It requires that two individuals belong to different social groups if at least one of them thinks so.

Negative Liberalism (NL): A group identity function $f$ satisfies Negative Liberalism if for every profile $\boldsymbol{\pi} \in \mathcal{D}$ and all $i, j \in N, i \notin \pi_{j}^{j}$ implies $f(\boldsymbol{\pi})_{i} \neq f(\boldsymbol{\pi})_{j}$.

As is easily seen, all social groups generated by any group identity function satisfying NL must form a clique as two individuals are put in the same social group only if they classify each other in the given profile as members of the same class. Formally, given a profile of individual partitions, a clique is a subset $D \subseteq N$ such that for all $i, j \in D, j \in \pi_{i}^{i}$. A trivial way to satisfy NL is to let the group identity function always select the partition into singletons, i.e., to never put two different individuals in the same group. The following condition represents a minimal requirement that prevents this; it can be seen as a positive counterpart of condition NGS.

Positive Group Sovereignty (PGS): A group identity function $f$ satisfies Positive Group Sovereignty if for no profile $\boldsymbol{\pi} \in \mathcal{D}$ there exist $j$ and $k$ such that $j \notin f(\boldsymbol{\pi})_{k}$ and $\{j\} \cup f(\boldsymbol{\pi})_{k} \subseteq \pi_{i}^{i}$ for all $i \in\{j\} \cup f(\boldsymbol{\pi})_{k}$.

Thus, PGS requires that the societal classification should not allow a situation in which an individual thinks he belongs to every single individual of a social group he is not a member of if all members of this group think they belong to this individual and moreover to any other member of the group. As is easily verified, PGS and NL jointly imply that the societal classification generated at any profile of individual views is a coarsest partition into cliques.

Notice that, analogously to the case of PL and NGS, conditions NL and PGS alone do not imply the non-bossiness condition NB either. To verify this, 
consider a situation where there are different coarsest partitions into cliques, for instance in the situation of Fig. 1 above these are the partitions $\pi=$ $\{\{1,2\},\{3\},\{4,5\},\{6\},\{7\},\{8\}\}$ and $\pi^{\prime}=\{\{1\},\{2,3\},\{4,5\},\{6\},\{7\},\{8\}\}$. Define a group identity function $f$ as follows: $f$ selects $\pi$ if the individual views w.r.t. their own classification are as shown in Fig. 1 and individual 6 thinks that individuals 1 and 2 belong together, $f$ selects $\pi^{\prime}$ if the individual views w.r.t. their own classification are as shown in Fig. 1 and individual 6 does not think that individuals 1 and 2 belong together, and $f$ selects some coarsest partition into cliques in any other case. Clearly, $f$ then satisfies NL and PGS but violates non-bossiness. The same example shows that NL, PGS, and SET do not imply NB either.

Finally, it turns out that no group identity function can jointly satisfy NL, PGS, and $\mathrm{ET}^{+}$. Let $N=\{1,2,3\}$ and $\pi^{1}=\{\{1,2\},\{3\}\}, \pi^{2}=\{\{1,2,3\}\}$, $\pi^{3}=\{\{1\},\{2,3\}\}$. For any group identity function $f$ satisfying NL and PGS one has $f(\boldsymbol{\pi}) \in\{\{\{1,2\},\{3\}\},\{\{1\},\{2,3\}\}\}$. If $f(\boldsymbol{\pi})=\{\{1,2\},\{3\}\}$, then $f$ grants the wish of individual 1 but not of individual 3. Similarly, if $f(\boldsymbol{\pi})=\{\{1\},\{2,3\}\}$, then $f$ grants the wish of individual 3 but not of individual 1. Hence, each selection violates $\mathrm{ET}^{+}$. The same example also shows that no aggregation rule satisfies $\mathrm{PGS}$ and $\mathrm{ET}^{+}$in combination of a weaker version of NL only requiring that two individuals should belong to different social groups if both of them think so.

\section{References}

[1] Barthélemy, J.P. (1988): Comments on: Aggregation of equivalence relations by P.C. Fishburn and A. Rubinstein, Journal of Classification 5, 
$85-87$.

[2] Barthélemy, J.P., B. Leclerc, and B. Montjardet (1986): On the use of ordered sets in problems of comparison and consensus of classifications, Journal of Classification 3, 187-224.

[3] Çengelci, M. and R. Sanver (2010): Simple collective identity functions, Theory and Decision 68(4), 417-443.

[4] Dimitrov, D., T. Marchant, and D. Mishra (2011): Separability and aggregation of equivalence relations, Economic Theory, forthcoming.

[5] Dimitrov, D., S.-C. Sung, and Y. Xu (2007): Procedural group identification, Mathematical Social Sciences 54, 137-146.

[6] Fishburn, P.C. and A. Rubinstein (1986): Aggregation of equivalence relations, Journal of Classification 3, 61-65.

[7] Houy, N. (2007): 'I want to be a J!": Liberalism in group identification problems, Mathematical Social Sciences 54, 59-70.

[8] Kasher, A. and A. Rubinstein (1997): On the question "Who is a J?" A social choice approach, Logique et Analyse 160, 385-395.

[9] Miller, A. (2008): Group identification, Games and Economic Behavior 63, 188-202.

[10] Mirkin, B. (1975): On the problem of reconciling partitions, in: Blalock, H.M., A. Aganbegian, F.M. Borodkin, R. Boudon, V. Capecchi (Eds.), Quantitative Sociology, International Perspectives on Mathematical and Statistical Modelling. Academic Press, New York, pp. 441-449.

[11] Satterthwaite, M. and H. Sonnenschein (1981): Strategy-proof allocation mechanisms at differentiable points, Review of Economic Studies $48,587-598$. 
[12] Samet, D. and D. Schmeidler (2003): Between liberalism and democracy, Journal of Economic Theory 110, 213-233. 


\section{Working Paper Series in Economics}

recent issues

No. 23 Dinko Dimitrov and Clemens Puppe: Non-bossy social classification, April 2011

No. 22 Kim Kaivanto and Eike B. Kroll: Negative recency, randomization device choice, and reduction of compound lotteries, April 2011

No. 21 Antje Schimke and Thomas Brenner: Long-run factors of firm growth - a study of German firms, April 2011

No. 20 Aaron B. Scholz: Spatial network configurations of cargo airlines, April 2011

No. 19 Arne Beck: Public bus transport in Germany - a proposal to improve the current awarding system, April 2011

No.18 Nina Menz and Ingrid Ott: On the role of general purpose technologies within the Marshall-Jacobs controversy: the case of nanotechnologies, April 2011

No. 17 Berno Buechel: A note on Condorcet consistency and the median voter, April 2011

No. 16 Eike B. Kroll, Jörg Rieger and Bodo Vogt: How performance based payoffs influence estimates of complex information? An experimental study on quality and precision in estimation tasks, March 2011

No. 15 Siegfried K. Berninghaus, Thomas Neumann and Bodo Vogt: Learning in networks - an experimental study using stationary concepts, February 2011

No. 14 Marten Hillebrand: Governmental debt, interest policy, and tax stabilization in a stochastic OLG economy, January 2011

No. 13 Arne Beck and Matthias Walter: Tender prices in local bus transport in Germany - an application of alternative regression techniques, December 2010 